\title{
Bursting neurons in the hippocampal formation convey information about LFP features
}

\author{
Maria Constantinou ${ }^{1 *}$, Daniel H Elijah ${ }^{1}$, Daniel Squirrell ${ }^{1}$, Inés Samengo ${ }^{2}$, John Gigg ${ }^{1}$, Marcelo A Montemurro ${ }^{1}$ \\ From The Twenty Third Annual Computational Neuroscience Meeting: CNS*2014 \\ Québec City, Canada. 26-31 July 2014
}

Local field potentials (LFPs) are thought to reflect the combined activity of neuronal populations projecting onto a local area. Accumulating evidence suggests that LFPs can contribute to neural information encoding by providing a reference signal that can boost the information conveyed by spikes $[1,2]$. Although some mechanisms have been proposed to explain how this extra information might be made available to downstream neurons $[3,4]$, they are yet to be tested against experimental data. In this study, we used both modelling of hippocampal pyramidal neurons and in-vivo data to investigate how neurons in the hippocampal formation can use bursting to convey information about features of LFPs. Intrinsic bursts produced by pyramidal neurons provide a graded signal determined by the burst spike count. Fitting a dual-compartment model of a pyramidal cell to realistic burst firing $[3,5,6]$ shows that bursts with spike counts of 1 (tonic firing), 2 and 3 or more are triggered with a differential locking with respect to features of the reference LFPs. This locking provides an explicit mechanism for bursts of different spike count to transmit information contained in LFPs to downstream neurons. We tested the model predictions by analysing recordings of spontaneous LFPs and spiking activity from areas CA1 and subiculum of urethane-anaesthetised rats. Statistical analyses and information theory were used to quantify the encoding capacity of bursts to discriminate basic features of LFPs, such as phase, slope, instantaneous voltage and energy. In agreement with model predictions, features such as phase and slope were found to be the most reliable single features correlated to burst size. We also applied multiple discriminant analysis to characterise the set of optimal LFP features that were maximally discriminated by different bursts. We found that burst spike count conveyed significantly more information about these optimal LFP features compared to simple LFP parameters. Our results provide the first in-vivo evidence that neurons can use bursts to convert information about LFP features into a spike-count code that is suitable to be decoded by postsynaptic neurons in distant neural networks.

\section{Authors' details}

${ }^{1}$ Faculty of Life Sciences, University of Manchester, Manchester, M13 9PT, UK. ${ }^{2}$ Centro Atómico Bariloche and Instituto Balseiro, San Carlos de Bariloche, 8400, Argentina.

Published: 21 July 2014

\section{References}

1. Montemurro MA, Rasch MJ, Murayama Y, Logothetis NK, Panzeri S: Phaseof-firing coding of natural visual stimuli in primary visual cortex. Curr Biol 2008, 18(5):375-380.

2. Kayser C, Montemurro MA, Logothetis NK, Panzeri S: Spike-phase coding boosts and stabilizes information carried by spatial and temporal spike patterns. Neuron 2009, 61(4):597-608.

3. Samengo I, Montemurro MA: Conversion of phase information into a spike-count code by bursting neurons. PLOS ONE 2010, 5(3):e9669.

4. Masquelier T, Hugues E, Deco G, Thorpe SJ: Oscillations, phase-of-firing coding, and spike timing-dependent plasticity: an efficient learning scheme. J Neurosci 2009, 29(43):13484-13493.

5. Kepecs A, Lisman J: Information encoding and computation with spikes and bursts. Network: Comput Neural Syst 2003, 14(1):103-118.

6. Pinsky PF, Rinzel J: Intrinsic and network rhythmogenesis in a reduced Traub model for CA3 neurons. J Comput Neurosci 1994, 1(1-2):39-60.

doi:10.1186/1471-2202-15-S1-P89

Cite this article as: Constantinou et al:: Bursting neurons in the hippocampal formation convey information about LFP features. BMC Neuroscience 2014 15(Suppl 1):P89. 